\title{
Quantify Prostate Cancer by Automated Histomorphometry
}

\author{
Ulf-Dietrich Braumann ${ }^{1}$, Jens-Peer Kuska ${ }^{2}$, Markus Löffler ${ }^{3}$, Nicolas Wernert ${ }^{4}$ \\ ${ }^{1}$ Translational Centre for Regenerative Medicine, University Leipzig, 04103 Leipzig \\ ${ }^{2}$ Interdisciplinary Centre for Bioinformatics, University Leipzig, 04107 Leipzig \\ ${ }^{3}$ Inst. for Medical Informatics, Statistics \& Epidemiology, Univ. Leipzig, 04107 Leipzig \\ ${ }^{4}$ Institute of Pathology, University Bonn, 53105 Bonn \\ ubraumann@trm. uni-leipzig.de
}

\begin{abstract}
A new method is presented to quantify malignant changes in histological sections of prostate tissue immunohistochemically stained for prostate-specific antigen (PSA) by means of image processing. The morphological analysis of the prostate tissue uses the solidity of PSApositive prostate tissue segments to compute a quantitative measure that turns out highly correlated with scores obtained from routine diagnosis (Gleason, Dhom).
\end{abstract}

\section{Introduction}

Prostate cancer is one of the most frequent cancers in western countries. Routine diagnosis of prostate cancer operates on several levels of resolution. With low microscopic magnification, the shape of the tissues is examined, while on large magnifications on the level of single cells the shape of the cell nucleus and the size of the individual cells is of interest. In the present paper we restrict ourselves to the low magnification scale and look for shape descriptors of the cancer tissue. While a healthy glandular tissue has a regular shape that reflects its function, the cancer tissue has lost this regular structure. We are interested in the quantification of this structural change in prostate cancer. Further, we wish to obtain a quantitative estimate of the cancer shape that compares well to the diagnostic grading obtained by a pathologist. The grading determines the future treatment of the patient, and a more objective measure than the opinion of the pathologist seems to be preferable. The Gleason grading [1] uses the degree of the differentiation of the glandular tissue and the pattern of the glands. The Dhom grading [2] uses additional cytological parameters. We here report on a new algorithm designed to quantify prostate glandular structures and applied this to 26 specimens of 23 patients. 


\section{Materials and Methods}

\subsection{Segmentation}

All images where preprocessed by a channel-wise total variation filter [3] in RGB space to reduce the spatial noise and obtain more compact regions in the segmentation.

It is typical for immunohistochemical stains that the color distribution may exhibit quite a wide variation in color saturation across different slides or even on the same slide. Our goal was to segment all images with the same set of parameters for the segmentation. For this, a c-means segmentation in the CIE $\mathrm{Lu}^{*} \mathrm{v}^{*}$ color space was chosen $[4,5]$. The result of an individual c-means segmentation are the segmented image and a set of colors that are used as centers in the clustering process. It is always difficult to select initial centers for the cmeans segmentation, so we take four centers (rough estimates) and make a first loop over all images that yield new centers for every image. These new centers are averaged over all processed images and taken as starting values for a second c-means segmentation over all images. The resulting segmentations were then used to select the class that corresponds to the prostate tissue in order to later on characterize the shape of the binarized segments. Fig. 1 depicts the result for three samples from the set with an overall of 96 regions of interest (ROIs) out of 26 specimens.

For every connected component (segment, i.e. glandular structure of interest, GOI) referring to a binary mask corresponding to the immunohistochemically stained prostate tissue we compute the solidity of each GOI [6]. The solidity of a single shape $i$ is defined as

$$
s_{i}=\frac{H_{i}}{A_{i}}
$$

where $H_{i}$ is the area of the convex hull of the component and $A_{i}$ is its real area (see Fig. 2).

For convex shapes like disks or rectangles the solidity is always equal to one. For non-convex objects one gets always values larger than one. The solidity is invariant under translation, rotation and scaling. To get a single number for every image we compute a weighted solidity

$$
S=\frac{\sum_{i} A_{i} s_{i}}{\sum_{i} A_{i}}=\frac{\sum_{i} H_{i}}{\sum_{i} A_{i}} .
$$

The summation runs over all $i$ components in the image. The weighting is used, since otherwise small objects should not contribute to the averaged solidity the same way as large objects do. By this averaging process we introduce the important quantity of the size into the calculation that otherwise would be lost by averaging the pure scale invariant solidity.

\section{$3 \quad$ Results}

To relate the computed averaged solidities with the gradings obtained from the diagnostic findings box-whisker plots are made from the mean solidity in every 


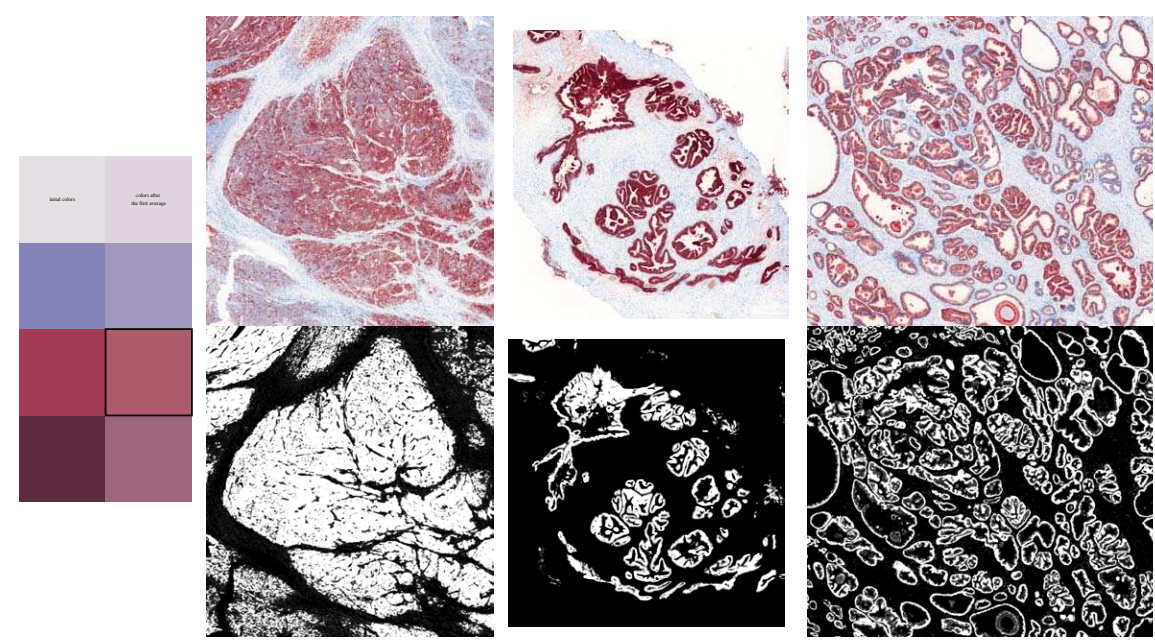

Fig. 1. Left: Colors used as initial centers for the c-means segmentation. That color corresponding to the prostate tissue color is marked by a black frame. Right: Three sample ROIs (upper row) and their segmentation (lower row)

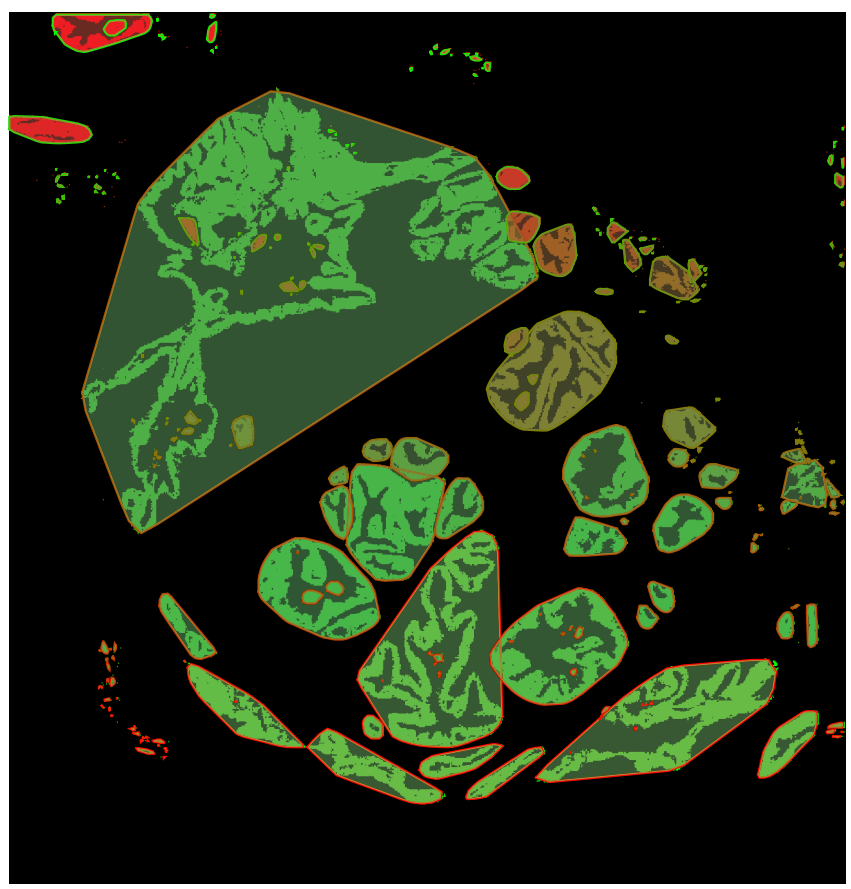

Fig. 2. Solidity for the components (GOIs) determined as ratio of the area of the component's convex hull to the real component's area. For every segmented component (GOI) within this sample ROI, its corresponding convex hull is overlayed in the same label color (shades of green, brown and red) but with $50 \%$ transparency 
Fig. 3. Box-whisker plots for the mean solidity of the 96 ROIs for Gleason scoring and Dhom grading. The value 0 in both grading systems was assigned cases without malign changes
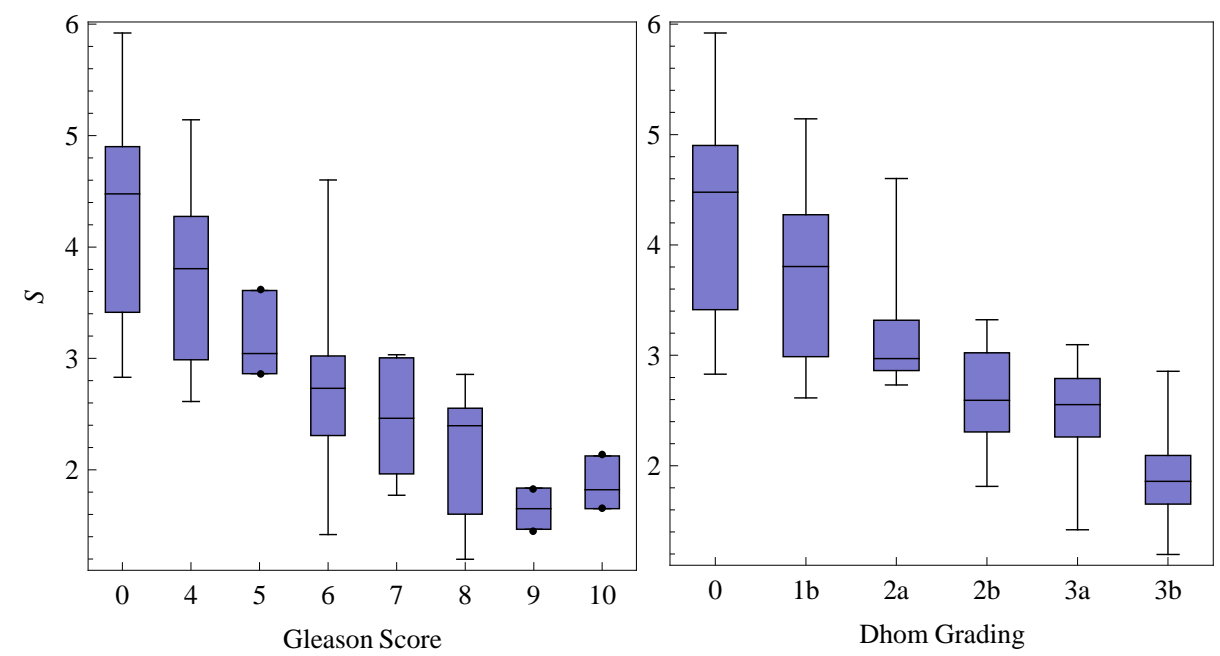

pathological grade class (Fig. 3). For both grading system we obtain a clear correlation of the computed solidities with the grading. Lower mean solidities correspond to higher scores in both grading systems.

\section{Discussion}

The developed segmentation procedure works well for a wide range of PSA stained samples and allows a robust detection of the PSA-positive prostatic tissue. The mean solidity seems to be a good objective measure for the degree of malignancy of the cancer. It should be noted that the above-mentioned established grading systems as typically are applied in routine pathology - although in good correspondence with our solidity-based histomorphometric assessments - are not considered as gold standards here. Rather, our work is driven by the need of a reliable, reproducible objective quantification and characterization of the malignancy of prostatic changes in order to provide a better basis for prognosis and therapy decisions. With our solidity-based automated histomorphometric approach we have introduced a simple but promising objective method ready for further assessments concerning its appropriateness to support diagnostic findings.

Acknowledgement. The project is supported by the Federal Ministry of Education and Research (BMBF), grant PTJ-BIO/31P4282 (MS CartPro) and grant PTJ-BIO/031390 (TRM Leipzig). 


\section{References}

1. Gleason DF. Classification of prostatic carcinomas. Cancer Chemotherapy Reports. 1966;50(3):125-8.

2. Helpap B, Böcking A, Dhom G, et al. Klassifikation, histologisches und zytologisches Grading sowie Regressionsgrading des Prostatakarzinoms. Eine Empfehlung des pathologisch-urologischen Arbeitskreises „Prostatakarzinom“. Pathologe. 1985;6:37.

3. Chan TF, Osher S, Shen J. The digital TV filter and nonlinear denoising. IEEE Trans Image Process. 2001;10(2):231-41.

4. Linde Y, Buzo A, Gray RM. An algorithm for vector quantizer design. IEEE Trans Commun. 1980;28(1):84-95.

5. Lloyd SP. Least squares quantization in PCM. IEEE Trans Inform Theory. 1982;28(2):129-37.

6. Gonzalez RC, Woods RE. Digital Image Processing. 2nd ed. Prentice Hall; 2002. 